\title{
The Winding Path Towards an Inverse Relationship Between Sun Exposure and All-cause Mortality
}

\author{
PELLE G. LINDQVIST \\ Clintec, Karolinska Institute, Stockholm, Sweden
}

\begin{abstract}
For a long time, skin cancer has been known to be related to extensive $U V$ exposure. New emerging data have, however, shown low UV exposure/low vitamin D levels to be related to increased mortality rate due to skin cancer. In addition, low sun exposure habits in regions of low solar intensity have been shown to be a major risk factor for allcause mortality in the same range as that for smoking. This is mainly due to lower all-cause mortality due to cardiovascular disease (CVD) and non-CVD/non-cancer disease among women with active sun exposure. Women with active sun exposure habits were estimated to have a 1- to 2year longer life-expectancy during the Melanoma in Southern Sweden study interval. These findings are in line with those to be expected from an evolutionary perspective and research findings, but in opposition to present guidelines and recommendations.
\end{abstract}

Most dietary products are low in vitamin D and sunlight is the main contributor to vitamin D status in humans. Ultraviolet B (UVB) radiation with a wavelength between 290 and $315 \mathrm{~nm}$ penetrates the skin and converts 7-dehydrocholesterol to 25hydroxycholecalciferol vitamin $\mathrm{D}_{3}$ via pre-vitamin $\mathrm{D}$ (1). The hydroxylation of 25-OH-vitamin $\mathrm{D}$ into its active form, $1 \mathrm{a}, 25(\mathrm{OH})_{2}$ vitamin $\mathrm{D}_{3}$, takes place mainly in the kidney (1). Vitamin D status is usually measured by the $25-\mathrm{OH}$-vitamin $\mathrm{D}$ level. There are different opinions regarding what are sufficient and deficient levels, and levels above $50 \mathrm{nmol} / 1$ 25-OH-vitamin D are usually regarded as sufficient (2).

This article is freely accessible online.

Correspondence to: Pelle G. Lindqvist, Clintec, Karolinska Institute, Sjukhusbacken 10, Plan 9, Sodersjukhuset, 11881 Stockholm, Sweden. Tel: +46 708992545, e-mail: pelle.lindqvist@ki.se

Key Words: Survival, mortality, UVB, sun, melanoma, CVD, review.
Both UV exposure and fair skin are major contributors to skin cancer. Non-melanoma skin cancer (NMSC) (i.e. basal cell carcinoma and squamous cell carcinoma) is mainly related to cumulative UV radiation and has a good prognosis. Malignant melanoma is related to episodic overexposure to UV radiation and increased mortality (3). Since heavy skin coloration is selected for close to the equator and fair skin coloration at higher latitudes there is speculation of an evolutionary advantage to having skin coloration appropriate for the regional sun intensity. However, evidence is lacking.

The aim of this review was to present how we came to the conclusion that there is an inverse relationship between sunexposure habits and all-cause mortality.

Venous thromboembolism (VTE) and sun exposure. There is a great demand for lifestyle advice from women at increased risk of VTE $(4,5)$. There have been reports of seasonal changes in VTE incidence, with a nadir in summer (6-8). In addition, vitamin $\mathrm{D}$ deficiency is an inflammatory condition and all inflammatory conditions increase the risk of VTE $(1,9)$. The Melanoma in Southern Sweden (MISS) cohort was drawn from the Swedish population registry by computerized random selection in 1990. Approximately 40,000 women with no history of cancer were invited to participate and 29,518 answered an initial written questionnaire. The questionnaire detailed sun exposure habits, aiming to identify those with extremely high sun exposure habits. With interest in the opposite, namely those with the lowest sun exposure habits, in whom vitamin D-deficient women would be over-represented, four questions were identified that could be used to identify those with low sun exposure habits: (i) How often do you sunbathe during the summertime? (never, 1-14 times, 15-30 times, $>30$ times); (ii) Do you sunbathe during the winter, such as on holiday to the mountains? (no; 1-3 days, 4-10 days; >10 days); (iii) Do you use tanning beds? (never; 1-3 times/year; 4-10 times/year; >10 times/year); and (iv) Do you go abroad on holiday to swim and sunbathe? (never; once/1-2 years; once/year; two or more times/year). The four questions were 
dichotomized into yes/no in the analysis (i.e. 'no/never' versus any other response). As a measure of total sun exposure, we created a four-score variable depending on the number of 'yes' responses to the above questions ranging from 0 (avoiding sun exposure: reference) to 4 (highest sun exposure).

All 312 women with VTE during the follow-up were identified. The analysis was adjusted for age, education, marital status, number of births, body mass index (BMI), and physical activity. For each increase in the number of 'no/never' responses to the sun exposure questions, the risk of having an incidental VTE increased by approximately $30 \%$. In addition, the risk in the winter months (December, January, and February) was $50 \%$ higher than that for other seasons.

Incidental type-2 diabetes mellitus (T2DM) and sun exposure. Individuals with T2DM were reported to have a lower vitamin D status and supplementation of $800 \mathrm{U}$ vitamin D daily lowered incidental T2DM (10-13). In addition, it was reported that vitamin D supplementation improved endothelial function in patients with T2DM with low vitamin D levels (14).

Based on the above, it was hypothesized that women with low sun exposure habits might be at an increased risk of incidental T2DM. The 537 women who were diagnosed after inception of the study during the 11-year follow-up were assessed. For each 'no' to the above sun exposure questions the risk of incidental T2DM was increased by $30 \%$. Adjustments for age, BMI, education parity, oral contraceptive use, smoking and exercise did not change risk estimates. However, in stratified analysis by BMI, lean women $\left(\mathrm{BMI}<25 \mathrm{~kg} / \mathrm{m}^{2}\right)$ had a $50 \%$ reduction of incidental T2DM for each 'no' to the four sun exposure questions.

Endometrial cancer and sun bed use. After an ecological study from Spain showed inverse correlation with latitude for endometrial cancer, it was hypothesized that low sun exposure habits might increase endometrial cancer risk (15). All 166 cases of endometrial cancer during the 15-year follow-up were identified and related to sun exposure habits. No significant relationship with sun exposure during summer or vacation was found. However, sun bed users were at a $40 \%$ significantly reduced risk of endometrial cancer (16).

Sun exposure and all-cause mortality. Since sun exposure showed strong dose-dependent inverse relationship with several of large diseases, it was hypothesized that sun exposure habits might be related to all-cause mortality. Furthermore, a Swedish study reported a reduced risk for all-cause mortality in women who went on sunbathing vacations more than once a year over three decades (17). One of the major criticisms from prior studies was that the result might be the effect of residual bias due to differences in income. Therefore, data on mean personalized income were added from the first four years after inception. There were 2545 deaths out of 29519 women who entered the study. Because there was interest in evaluating both differences in mortality rate with attained age as timescale and relative risk, flexible parametric models was chosen for survival analysis. Adjustments were done for disposable income, comorbidity at inception, age, smoking habits, education level, and marital status. The same sun exposure questions were used, but for presentation in Figure 1, sun exposure habits were categorized into three groups: zero 'yes' responses (avoidance of sun exposure); a 'yes' response to one or two questions (moderate sun exposure); and a 'yes' response to three or four questions (highest sun exposure).

There were highly significant differences between all three sun exposure groups in the relative risk estimations (18). As compared to the highest sun exposure group, the mortality rate was doubled [hazard ratio $(\mathrm{HR})=2.0,95 \%$ confidence interval $(\mathrm{CI})=1.6-2.5]$ among avoiders of sun exposure and increased by $40 \%(\mathrm{HR}=1.4,95 \% \mathrm{CI}=1.1-1.7)$ in those with moderate exposure when assessed with attained age as time variable. The population attributable risk for mortality for the group avoiding sun exposure was estimated to be $3 \%$.

All-cause mortality is usually divided into three major causes: cancer, cardiovascular disease (CVD), and nonCVD/non-cancer disease (19). If mortality is reduced in one of these, the proportion of deaths in the others will naturally increase. Differences in main causes of death due to different levels of sun exposure were investigated by a competing risk analysis. Subdistribution Cox regression analysis showed that for CVD death, avoidance of sun exposure was related to a $60 \%$ increased risk of death and the relationship was dose-dependent with subdistributional HR (sHR) $=1.46(95 \%$ $\mathrm{CI}=1.2-1.8)$ and $\mathrm{sHR}=2.3(95 \% \mathrm{CI}=1.8-3.1)$, for moderate and highest sun exposure groups, respectively. The corresponding sHR data for death due to non-CVD/noncancer were 1.57 (95\% $\mathrm{CI}=1.3-1.9)$ and 2.1 (95\% $\mathrm{CI}=1.7$ $2.8)$ and for death due to cancer $1.1(95 \% \mathrm{CI}=0.9-1.4)$ and 1.4 (95\% CI=1.04-1.6) respectively (19). The main findings of this analysis are displayed in Figure 2: the cumulative probability of death due to CVD, cancer, and non-CVD/noncancer death by sun exposure groups. The three bottom graphs show the relative contribution of CVD, cancer, and non-CVD/non-cancer to the all-cause mortality (19).

Thus, since life expectancy increased mainly for CVD and non-CVD/non-cancer, the relative contribution of death due to cancer increased with increasing sun exposure. Differences in life expectancy were assessed depending on sun exposure habit stratified by smoking in Figure 3. It can visually be seen that non-smokers who avoided sun exposure had a similar life expectancy as smokers with the most active sun exposure (Figure 3) (19). Thus, avoidance of sun exposure seemed to be a risk factor of a similar magnitude as smoking in terms of life expectancy. Differences in mean survival were assessed by restricted mean survival, i.e. the area under the 

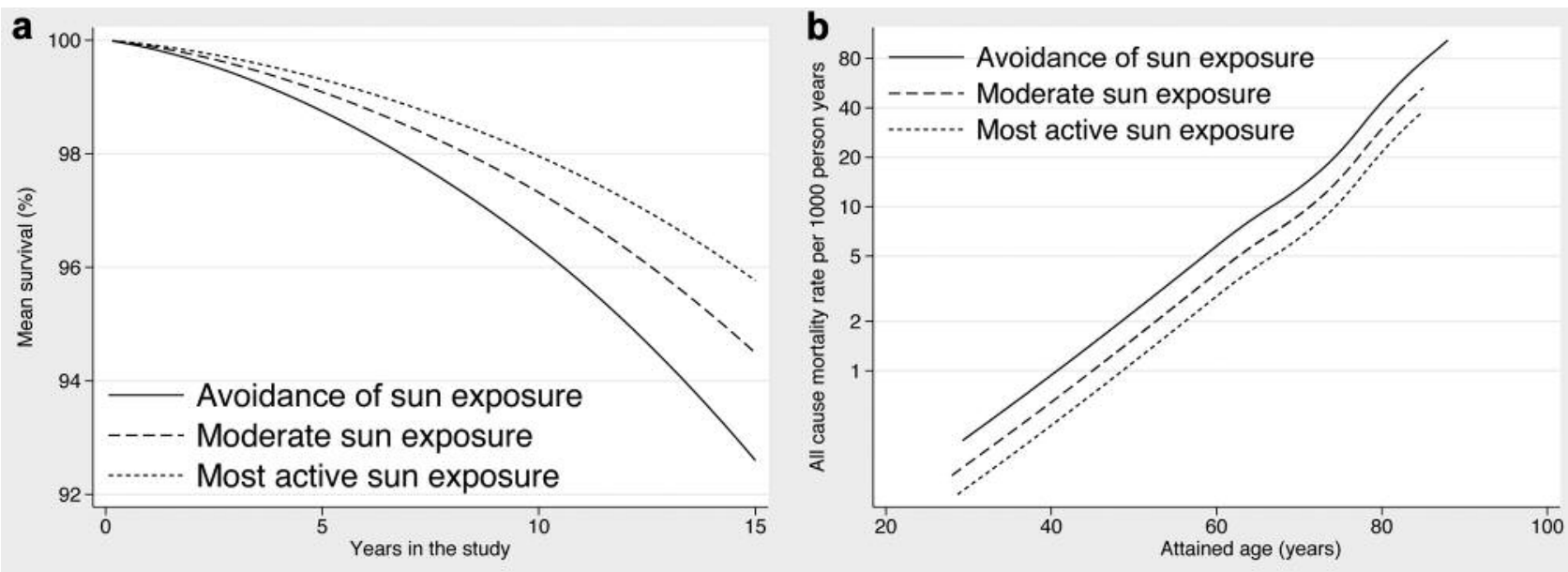

Figure 1. Mean survival (a) and all-cause mortality rate with attained age as time variable (b) by sun exposure habit. Sun exposure habits were categorized into three groups according to responses to a questionnaire on sun exposure habit: avoidance of sun exposure: zero 'yes' responses; moderate sun exposure: a 'yes' response to one or two questions; and most active sun exposure: a 'yes' response to three or four questions.
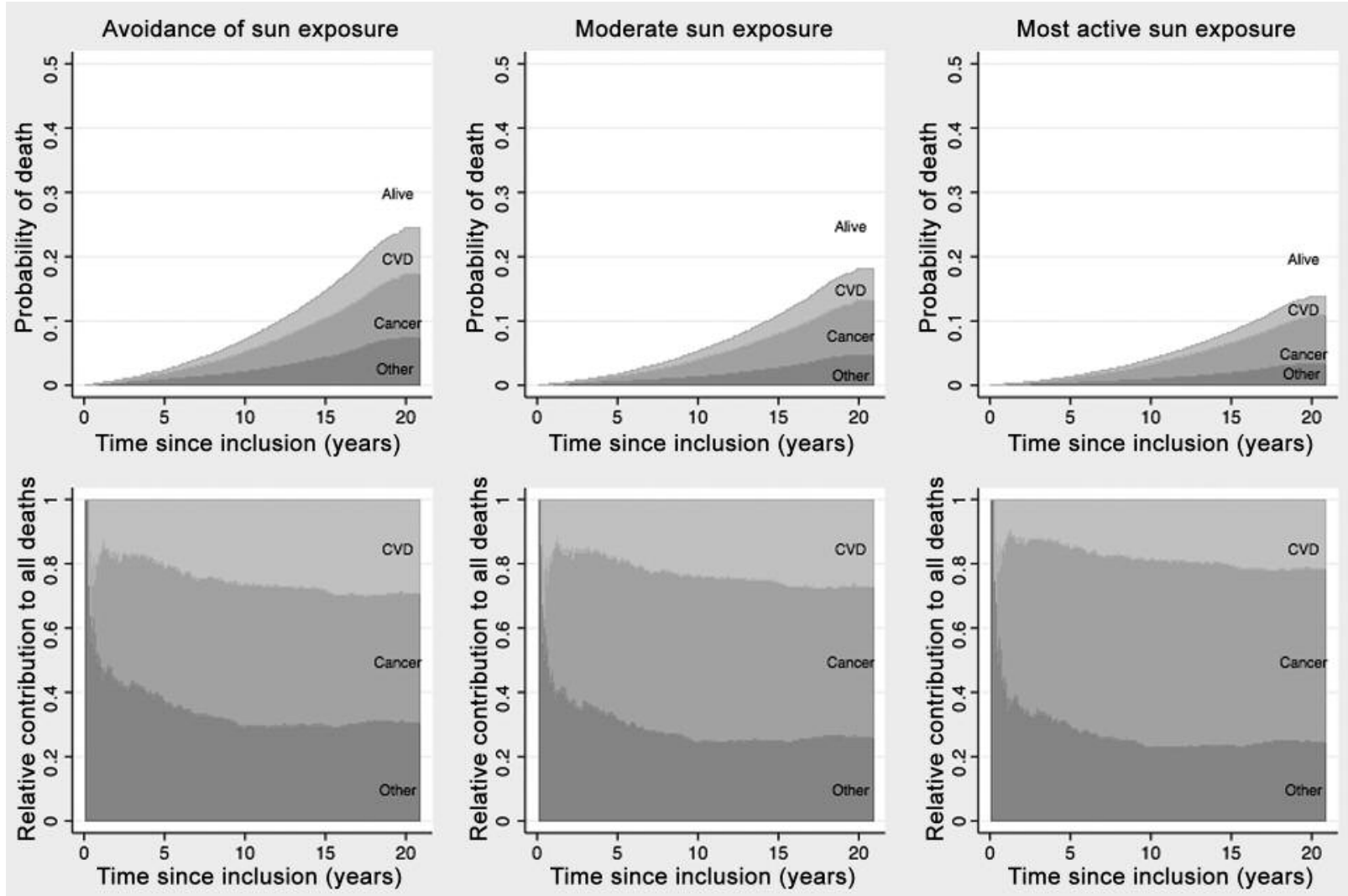

Figure 2. All-cause mortality in a competing risk scenario stratified by sun exposure habits, divided into three major causes. Sun exposure habits were categorized into three groups according to responses to a questionnaire on sun exposure habit: avoidance of sun exposure: zero 'yes' responses; moderate sun exposure: a 'yes' response to one or two questions; and most active sun exposure: a 'yes' response to three or four questions. With increasing sun exposure, the risk of all-cause death due to cardiovascular disease (CVD) and other (non-CVD/non-cancer) diseases is reduced (upper panel). The increased survival in those with CVD and other diseases results in increased prevalence of death due to cancer (lower panel). Age 55-64 at inclusion, no comorbidities, non-smokers, moderate disposable income. 


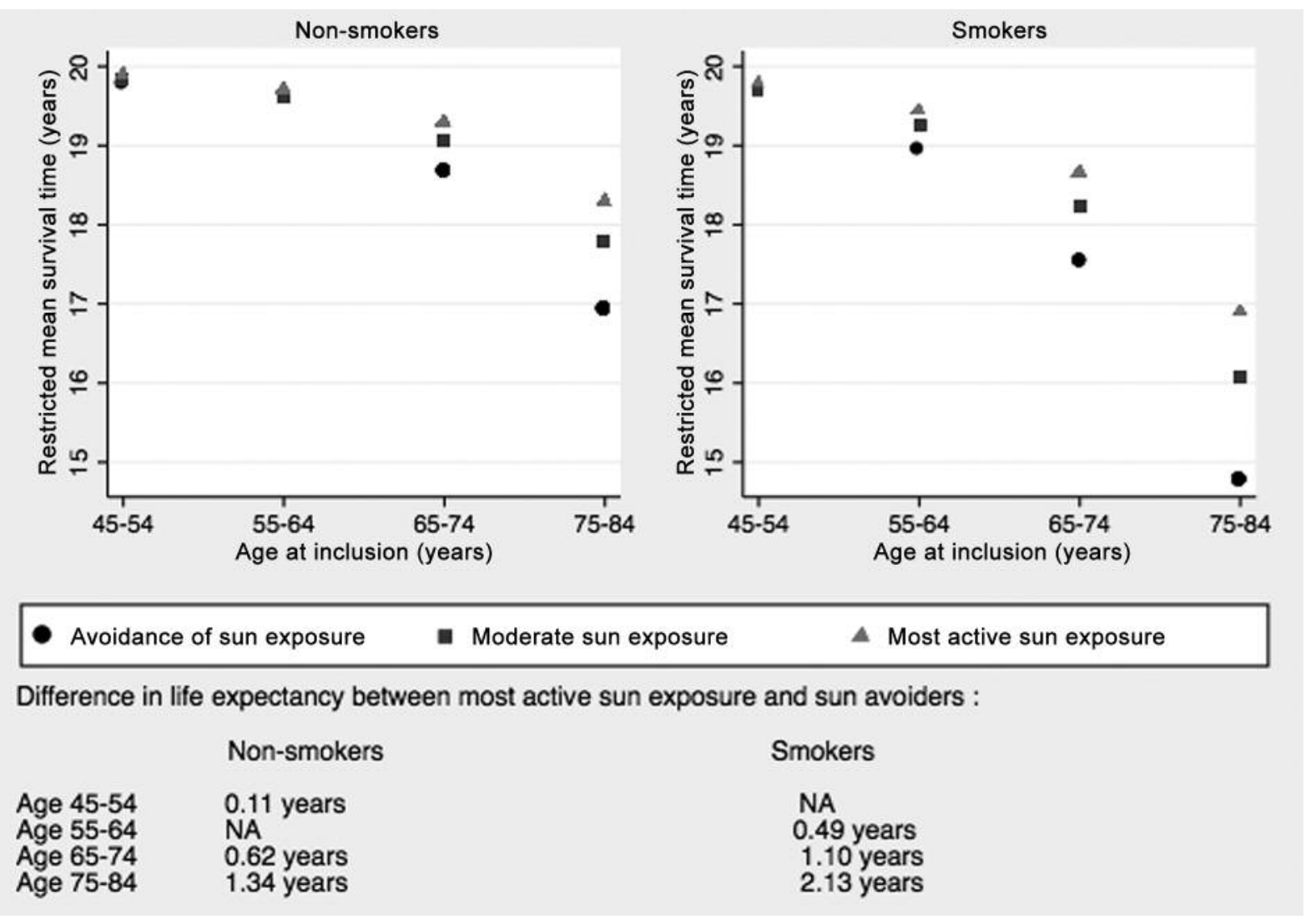

Figure 3. Difference in mean life expectancy according to sun exposure stratified for smoking. Sun exposure habits were categorized into three groups according to responses to a questionnaire on sun exposure habit: avoidance of sun exposure: zero 'yes' responses; moderate sun exposure: a 'yes' response to one or two questions; and most active sun exposure: a 'yes' response to three or four questions. The Figure show that nonsmokers avoiding sun exposure have a similar death rate as smokers with the greatest sun exposure, i.e. smoking and avoidance of sun exposure are risk factors of the same magnitude for death.

survival curve between two time points. For example, nonsmokers and smokers aged 60 years avoiding sun exposure had 1.3- and 2.1-year shorter life expectancy, respectively, as compared to those with the greatest sun exposure (19).

Skin cancer, sun exposure and all-cause mortality. There is a relationship between episodic high sun exposure and cutaneous melanoma incidence, but an inverse relationship to its prognosis (20). Thus, high UV exposure increases the incidence, while low sun exposure habits/vitamin D levels have been linked to thicker, more aggressive melanomas associated with shorter survival times (20-22). In line with these prior results, out of those developing melanoma during the study period, $35 \%$ of women with low sun exposure and $10 \%$ of those with the highest sun exposure habits died during the follow-up, i.e. an eight-fold increased risk of all-cause mortality among those with low sun exposure (19). Furthermore, when grouping women based on skin cancer status (no skin cancer, NMSC, or melanoma) and sun exposure habits (low sun exposure, moderate exposure, or highest exposure) (i.e. a $3 \times 3$ table), women developing NMSC with the greatest sun exposure habits had the lowest mortality rate, while those with melanoma and with low sun exposure (answered no on all four questions) had the greatest mortality rate (19).

For 40 years, there have been strong recommendations to avoid sun exposure due to risk of skin cancer, especially in those who are fair skinned. From an evolutionary perspective, human skin coloration seems to be modified depending on regional UV exposure. If sun exposure is important for human life and there are very few hours of sunlight, fair-skinned individuals might need a shorter time in the sun to achieve sufficiency, while UV exposure might not be sufficient for those with more heavy skin pigmentation. Future work should try to further reduce possible residual bias by a nested randomized controlled observational design comparing UV-sensitive to non-UV- 
sensitive individuals. Sensible sun exposure habits should be recommended. In 2014, there was one day in Sweden when the UV index was 6 (i.e. high), while for 8-9 months of the year it is low (UV index $<3$ ). Thus, in a country with scarcity of UV radiation and hardly any days for strong sun exposure, daily, short, sun exposure at midday could be recommended. The increasing incidence of melanoma in Sweden might be due to the reliance on sun screen use, which might have resulted in overexposure. No study has yet shown that it is safe to be out longer in the sun with sun blocker with regards to risk of melanoma than without sun blocker.

Maternal mortality. One of the largest causes of maternal mortality in Western countries is VTE (see above). Preeclampsia/eclampsia is a major cause of maternal mortality worldwide (23). Eclampsia has been shown to be more prevalent during the winter (24). Earlier, it was hypothesized that this was due to cold or humidity, but we recently suggested an alternative interpretation that lack of sun exposure might be the environmental factor causing the increased risk of eclampsia (25). Vitamin D deficiency in late pregnancy is related to an increased risk of pre-eclampsia (26). Results from early pregnancy are more heterogeneous (27). Thus, our interpretation is that pre-eclampsia is not caused by vitamin D deficiency, but that its deficiency may aggravate the condition.

Perinatal death. Vitamin D has an immunomodulatory, antiinflammatory effect and produces antibacterial proteins, and might be involved in the etiology of both preterm birth and infections $(26,28)$. Furthermore, vitamin D deficiency has been associated with fetal growth restriction, a major cause of perinatal death. Vitamin D deficiency is also related to altered muscle strength, including fetal cardiac muscle strength (1). Thus, fetuses of vitamin D-deficient mothers might be more vulnerable to the hypoxic stress of delivery. Recently, it was shown that women with vitamin D deficiency in early pregnancy were more likely to undergo emergency cesarean delivery due to suspected fetal distress and the risk of newborn asphyxia was doubled (29).

Strengths and limitations. The large sample size drawn from the population registry is a strength of the MISS study. In addition, since data were collected at study inception, there was no risk of recall bias. The major drawback is that it is not possible to separate a healthy lifestyle from an active sun exposure habit. Findings were based on observational findings and might not be causal. However, the strong dosedependency increases the likelihood of causality.

Prevention. We know that women with active sun exposure habits have lower risk of CVD and non-CVD/non-cancer disease. Thus, sensible sun exposure habits should be recommended. We do not yet know by what mechanism sun exposure reduces mortality (30). Avoidance of vitamin D deficiency is one of the main hypotheses, and vitamin D supplementation could be used to achieve this. However, controlled studies of vitamin D supplementation do not show the same positive picture as observational studies, indicating that other mechanisms might be present (30).

\section{Conclusion}

Observational data indicate a strong inverse relationship between all-cause mortality and sun exposure. Data indicate that low sun exposure mainly increases the risk of CVD and non-CVD/non-cancer disease. The introduction of sensible sun exposure recommendations might improve public health. Due to the high prevalence of vitamin D deficiency, pregnant women should be recommended vitamin D supplementation.

\section{References}

1 Holick MF: Vitamin D deficiency. N Engl J Med 357: 266-281, 2007.

2 Ross AC, Manson JE, Abrams SA, Aloia JF, Brannon PM, Clinton SK, Durazo-Arvizu RA, Gallagher JC, Gallo RL, Jones G, Kovacs CS, Mayne ST, Rosen CJ and Shapses SA: The 2011 report on dietary reference intakes for calcium and vitamin D from the Institute of Medicine: What clinicians need to know. J Clin Endocrinol Metab 96: 53-58, 2011.

3 Reszko A, Aasi SZ, Wilson LD and Leffel DJ: Cancer of the Skin. In: Cancer Principles \& Practice of Oncology. Volume 2. DeVita VT, Lawrence TS, Rosenberg SA, DePinho RA and Weinberg RA (eds). Philadelphia: Lippincott Williams \& Wilkins, 2011.

4 Lindqvist PG, Svensson PJ, Marsaal K, Grennert L, Luterkort M and Dahlback B: Activated protein C resistance (FV:Q506) and pregnancy. Thromb Haemost 81: 532-537, 1999.

5 Lindqvist PG and Dahlback B: Reactions to awareness of activated protein $\mathrm{C}$ resistance carriership: a descriptive study of 270 women. Acta Obstet Gynecol Scand 82: 467-470, 2003.

6 Gallerani M, Boari B, Smolensky MH, Salmi R, Fabbri D, Contato E and Manfredini R: Seasonal variation in occurrence of pulmonary embolism: analysis of the database of the EmiliaRomagna region, Italy. Chronobiol Int 24: 143-160, 2007.

7 Gallerani M, Boari B, de Toma D, Salmi R and Manfredini R: Seasonal variation in the occurrence of deep vein thrombosis. Med Sci Monit 10: CR191-196, 2004.

8 Clauss R, Mayes J, Hilton P and Lawrenson R: The influence of weather and environment on pulmonary embolism: pollutants and fossil fuels. Med Hypotheses 64: 1198-1201, 2005.

9 Blondon M, Cushman M, Jenny N, Michos ED, Smith NL, Kestenbaum B and de Boer IH: Associations of serum 25hydroxyvitamin D with hemostatic and inflammatory biomarkers in the Multi-Ethnic Study of Atherosclerosis. J Clin Endocrinol Metab 101: 2348-2357, 2016.

10 Knekt P, Laaksonen M, Mattila C, Harkanen T, Marniemi J, Heliovaara M, Rissanen H, Montonen J and Reunanen A: Serum vitamin $\mathrm{D}$ and subsequent occurrence of type 2 diabetes. Epidemiology 19: 666-671, 2008. 
11 Scragg R, Sowers M and Bell C: Serum 25-hydroxyvitamin D, diabetes and ethnicity in the Third National Health and Nutrition Examination Survey. Diabetes Care 27: 2813-2818, 2004.

12 Pittas AG, Dawson-Hughes B, Li T, Van Dam RM, Willett WC, Manson JE and Hu FB: Vitamin D and calcium intake in relation to type 2 diabetes in women. Diabetes Care 29: 650-656, 2006.

13 Pittas AG, Lau J, Hu FB and Dawson-Hughes B: The role of vitamin $\mathrm{D}$ and calcium in type 2 diabetes. A systematic review and meta-analysis. J Clin Endocrinol Metab 92: 2017-2029, 2007.

14 Sugden JA, Davies JI, Witham MD, Morris AD and Struthers AD: Vitamin D improves endothelial function in patients with Type 2 diabetes mellitus and low vitamin D levels. Diabet Med 25: 320-325, 2008.

15 Grant WB: An ecologic study of cancer mortality rates in Spain with respect to indices of solar UVB irradiance and smoking. Int J Cancer 120: 1123-1128, 2007.

16 Epstein E, Lindqvist PG, Geppert B and Olsson H: A populationbased cohort study on sun habits and endometrial cancer. Br J Cancer 101: 537-540, 2009.

17 Yang L, Lof M, Veierod MB, Sandin S, Adami HO and Weiderpass E: Ultraviolet exposure and mortality among women in Sweden. Cancer Epidemiol Biomarkers Prev 20: 683-690, 2011.

18 Lindqvist PG, Epstein E, Landin-Olsson M, Ingvar C, Nielsen $\mathrm{K}$, Stenbeck $\mathrm{M}$ and Olsson $\mathrm{H}$ : Avoidance of sun exposure is a risk factor for all-cause mortality: results from the Melanoma in Southern Sweden cohort. J Intern Med 276: 77-86, 2014.

19 Lindqvist PG, Epstein E, Nielsen K, Landin-Olsson M, Ingvar $\mathrm{C}$ and Olsson $\mathrm{H}$ : Avoidance of sun exposure as a risk factor for major causes of death: a competing risk analysis of the Melanoma in Southern Sweden cohort. J Intern Med 280(4): 375-387, 2016.

20 Newton-Bishop JA, Beswick S, Randerson-Moor J, Chang YM, Affleck P, Elliott F, Chan M, Leake S, Karpavicius B, Haynes S, Kukalizch K, Whitaker L, Jackson S, Gerry E, Nolan C, Bertram C, Marsden J, Elder DE, Barrett JH and Bishop DT: Serum 25-hydroxyvitamin $\mathrm{D}_{3}$ levels are associated with breslow thickness at presentation and survival from melanoma. J Clin Oncol 27: 5439-5444, 2009.
21 Masback A, Westerdahl J, Ingvar C, Olsson H and Jonsson N: Clinical and histopathological characteristics in relation to aetiological risk factors in cutaneous melanoma: a populationbased study. Melanoma Res 9: 189-197, 1999.

22 Moan J, Baturaite Z, Porojnicu AC, Dahlback A and Juzeniene A: UVA, UVB and incidence of cutaneous malignant melanoma in Norway and Sweden. Photochem Photobiol Sci 11: 191-198, 2012.

23 Moodley J: Maternal deaths due to hypertensive disorders in pregnancy: Saving Mothers report 2002-2004. Cardiovasc J Africa 18: 358-361, 2007.

24 Rylander A and Lindqvist PG: Eclampsia is more prevalent during the winter season in Sweden. Acta Obstet Gynecol Scand 90: 114-117, 2011

25 Subramaniam V: Seasonal variation in the incidence of preeclampsia and eclampsia in tropical climatic conditions. BMC Womens Health 7: 18, 2007.

26 Triunfo S and Lanzone A: Potential impact of maternal vitamin D status on obstetric well-being. J Endocrinol Invest 39: 37-44, 2016.

27 Gidlof S, Silva AT, Gustafsson S and Lindqvist PG: Vitamin D and the risk of pre-eclampsia - a nested case-control study. Acta Obstet Gynecol Scand 94: 904-908, 2015.

28 Hulthen Varli I, Kublickas M, Papadogiannakis N and Petersson $\mathrm{K}$ : Chorioamnionitis without foetal inflammatory response is associated with stillbirth in early preterm pregnancies. J Matern Fetal Neonatal Med 26: 953-959, 2013.

29 Lindqvist PG, Silva AT, Gustafsson SA and Gidlof S: Maternal vitamin D deficiency and fetal distress/birth asphyxia: a population-based nested case-control study. BMJ open 6: e009733, 2016.

30 Lindqvist PG: Cardiology Patient Page. On the possible link between vitamin D deficiency and cardiovascular disease: Should we D-lighten our lives? Circulation 129: e413-414, 2014.

Received November 9, 2017

Revised December 25, 2017

Accepted January 1, 2018 